\title{
Genomic and clinical characterisation of multidrug-resistant carbapenemase- producing ST231 and ST16 Klebsiella pneumoniae isolates colonising patients at Siriraj hospital, Bangkok, Thailand from 2015 to 2017
}

Adhiratha Boonyasiri ${ }^{1,2^{*}}$, Elita Jauneikaite ${ }^{2,3}$, Lauren M. Brinkac ${ }^{4,5}$, Chris Greco ${ }^{4}$, Kanokorn Lerdlamyong ${ }^{6}$, Teerawit Tangkoskul ${ }^{6}$, Kevin Nguyen ${ }^{4}$, Visanu Thamlikitkul ${ }^{1,6}$ and Derrick E. Fouts ${ }^{4^{*}}$ (i)

\begin{abstract}
Background: Infections caused by carbapenemase-producing Enterobacteriaceae (CPE) have continually grown as a global public health threat, with significant mortality rates observed across the world. We examined the clinical data from patients with CPE infections and their outcomes, concentrating on Klebsiella pneumoniae isolates. We analysed the clinical information, performed antimicrobial susceptibility testing, and conducted molecular epidemiological and genomic analyses on the isolates to identify patterns in the data.

Methods: The clinical characteristics of 33 hospitalised patients with confirmed CPE, including patient-related factors associated with the development of CPE infections, were examined. Patients were divided according to whether they were "colonised" or "infected" with CPE and by the timing and frequency of their rectal swab collections, from which 45 swabs were randomly selected for analysis. CPE isolates were purified, and antimicrobial susceptibility tests performed. Whole genome sequences of these isolates were determined and analysed to compute bacterial multilocus sequence types and plasmid replicon types, infer phylogenetic relationships, and identify antimicrobial resistance and virulence genes.

(Continued on next page)
\end{abstract}

\footnotetext{
* Correspondence: ab11915@ic.ac.uk; dfouts@jcvi.org

${ }^{1}$ Department of Research and Development, Faculty of Medicine, Siriraj

Hospital, Mahidol University, Bangkok, Thailand

${ }^{4} \mathrm{~J}$. Craig Venter Institute, Rockville, MD, USA

Full list of author information is available at the end of the article
}

C C The Author(s). 2021 Open Access This article is licensed under a Creative Commons Attribution 4.0 International License, which permits use, sharing, adaptation, distribution and reproduction in any medium or format, as long as you give appropriate credit to the original author(s) and the source, provide a link to the Creative Commons licence, and indicate if changes were made. The images or other third party material in this article are included in the article's Creative Commons licence, unless indicated otherwise in a credit line to the material. If material is not included in the article's Creative Commons licence and your intended use is not permitted by statutory regulation or exceeds the permitted use, you will need to obtain permission directly from the copyright holder. To view a copy of this licence, visit http://creativecommons.org/licenses/by/4.0/ The Creative Commons Public Domain Dedication waiver (http://creativecommons.org/publicdomain/zero/1.0/) applies to the data made available in this article, unless otherwise stated in a credit line to the data. 


\begin{abstract}
(Continued from previous page)
Results: Altogether, 88.9\% (40/45) of the CPE isolates were K. pneumoniae. The most abundant carbapenemase gene family in the $K$. pneumoniae isolates $(33 / 39)$ was bla $a_{\mathrm{OXA}-232}$, with bla $a_{\mathrm{NDM}-1}$ additionally identified in 19 of them. All CPE isolates carrying either bla $a_{\mathrm{OXA}-232}$ or bla $a_{\mathrm{NDM}-1}$ were resistant to meropenem, but only 40 from 45 were susceptible to colistin. Among the CPE-infected patients $(n=18)$ and CPE-colonised patients who developed CPE infections during the study $(n=3)$, all but one received standard colistin-based combination therapy. Phylogenetic analysis revealed the polyclonal spread of carbapenemase-producing $K$. pneumoniae (CPKP) within the patient population, with the following two major subclades identified: ST16 $(n=15)$ and ST231 $(n=14)$. CPKP-ST231 had the highest virulence score of 4 and was associated with primary bacteraemia. The siderophores yersiniabactin and aerobactin, considered to be important virulence factors, were only identified in the CPKP-ST231 genomes.

Conclusions: This study has revealed the genomic features of colonising CPE isolates, focusing on antimicrobial resistance and virulence determinants. This type of multi-layered analysis can be further exploited in Thailand and elsewhere to modify the regimes used for empirical antibiotic treatment and improve the management strategies for CPE infections in hospitalised patients.
\end{abstract}

Keywords: Klebsiella pneumoniae isolates, Carbapenemase-producing Enterobacteriaceae, Antimicrobial resistance genes, Patient outcomes, Virulence genes, Phylogenetics

\section{Background}

Infections caused by carbapenemase-producing Enterobacteriaceae (CPE), which have increased worldwide in number to become a significant clinical problem over the last decade, are associated with high morbidity and mortality [1]. An early longitudinal study from Asia (2000-2012) revealed the prevalence of CPE was extremely low with average rates between $0.6-0.9 \%$ [2]. However, a survey from the National Antimicrobial Resistance Surveillance, Thailand from 2008 to 2016 revealed that carbapenemase-producing Klebsiella pneumoniae (CPKP) had increased in prevalence from $0.4 \%$ in 2008 to $5.4 \%$ in 2016 [3]. A recent retrospective cohort study from a 1200-bed university hospital in Bangkok reported on an increased incidence of CPE from 3.37 per 100,000 patient-days in 2011 to 32.49 per 100,000 patient-days between 2011 and 2016 [4]. The resistance mechanism for $\mathrm{CPE}$ is attributed to the following Ambler molecular classes of carbapenemhydrolysing beta-lactamases: class A (KPC), class B (IMP, NDM, VIM), and class D (OXA-48) [5]. There is insufficient data from Thailand on the distribution of beta-lactamase (bla) genes [6, 7]. However, a recent study from a university hospital in Bangkok revealed that $b l a_{\mathrm{NDM}}$ was the most common such gene followed by $b l a_{\text {OXA-48-like }}$ alleles (e.g., $b l a_{\text {OXA-48, }}, b l a_{\text {OXA-181 }}$, and

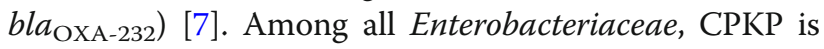
commonly associated with numerous antimicrobial resistance (AMR) genes and virulence determinants [8]. CPKP with its plasmid-encoded carbapenemases (e.g., $b l a_{\mathrm{NDM}}$ and $\left.b l a_{\mathrm{KPC}}\right)$ and its multiclass antibiotic resistance is associated with hospital-acquired infections and treatment challenges $[9,10]$. Hypervirulent $K$. pneumoniae (hvKP) can also cause invasive diseases such as liver abscesses and metastatic infections [11]. In addition to having the K1 capsular serotype, hvKP encodes other virulence determinants (e.g., yersiniabactin, aerobactin, and salmochelin siderophores), and rmpA1/rmpA2 genes, which upregulate capsule expression and are associated with more invasive infections $[8,12,13]$.

Although genetic diversity in carbapenemases has previously been reported in Thailand $[7,14]$, the epidemiology and characteristics of CPKP and its virulence determinants are not as well understood. Information is also lacking on the role played by hypervirulent strains of CPKP in hospital-acquired infections and how specific virulence determinants are associated with AMR profiles, disease severity and outcomes among hospitalised patients. The aim of the present study was to investigate the molecular epidemiological features of CPKP and its association with the clinical presentations of CPKPinfected patients. We also aimed to identify virulence determinants in the CPKP strains isolated from patients in this study.

\section{Methods}

Patient population and specimen collection

Eligible patients included all hospitalised patients aged $\geq 18$ years who had CPE recovered from clinical specimens submitted to the microbiology laboratory. The participants were classified as CPE-colonised patients or CPE-infected patients at enrolment. CPE-colonised patients were defined according to whether or not $\mathrm{CPE}$ carriage was found, but its presence was not associated with any symptoms or clinical disease. CPE-infected patients were defined according to whether or not CPE isolates were found at sites of infection diagnosed as $\mathrm{CPE}$ infections by clinicians. Rectal swabs were collected from the patients within $48 \mathrm{~h}$ of enrolment and then once a week until the specimens were CPE-negative for 
3 consecutive weeks (at the time of study there was no routine $\mathrm{CPE}$ screening procedure). One hundred and nineteen patients met the eligibility criteria, including 69 CPE-colonised patients and $50 \mathrm{CPE}$-infected patients at enrolment. Forty-five randomly selected and deidentified rectal swab samples from 33 patients, who were admitted for treatment between December 2015 and April 2017, were used for bacterial isolation and subsequent whole genome sequencing. Clinical information from each patient was collected, including information on patient demographics, clinical diagnosis, as well as their treatment during hospitalisation and outcome. The clinical outcomes of the CPE-infected patients at the end of treatment were classified as either 'favourable response' (absence or improvement of all clinical signs and symptoms of CPE infection) or 'unfavourable response' (worsening or persistence of clinical signs and/or symptoms of CPE infection, superinfection, or death).

\section{Bacterial isolates and antimicrobial susceptibility testing} Rectal swabs were inoculated onto MacConkey agar (BD, USA) supplemented with ceftriaxone $(4 \mathrm{mg} / \mathrm{L})$ and the plates incubated at $37^{\circ} \mathrm{C}$ for $18 \mathrm{~h}$. Bacterial identification was performed using Biotyper MALDI-TOF MS (Bruker Daltonics, Germany) according to the manufacturer's protocol. Colonies identified as Enterobacteriaceae were tested for antimicrobial susceptibility using standard methods and following the guidelines for the disk-diffusion method [15]. Confirmation of suspected carbapenemase production in Enterobacteriaceae-positive specimens was performed using a modified carbapenem inactivation method [16]. Phenotypic screening for the presence of carbapenemases was performed using a double-disc synergy approach with phenylboronic acid or ethylenediaminetetraacetic acid with meropenem as previously described [17]. Colistin resistance was tested using the broth microdilution method with cationadjusted Mueller-Hinton II broth [16]. Susceptibility to tigecycline was not tested at Siriraj Hospital during the study period.

\section{Genome sequencing and analysis}

DNA was extracted using the Wizard ${ }^{\circ}$ Genomic DNA Purification Kit (Promega, USA) and the multiplexed Illumina NexteraXT-generated libraries prepared from it were sequenced on the NextSeq 500 platform (Illumina, USA) using a $2 \times 150$-bp paired-end kit with an average target coverage of 100 -fold. Each read set was de novo assembled using SPAdes [18] and annotated with NCBI's prokaryotic genome annotation pipeline (PGAP) [19] as described previously [20, 21]. For phylogenetic analysis, reads were mapped to the $K$. pneumoniae QS17-0029 (GCA_003073235.1) reference genome, which was previously identified in Thailand and known to carry mor-1
[22], using Snippy v3.0 (https://github.com/tseemann/ snippy). FastTree v2.1 [23] was used to generate an approximate maximum-likelihood phylogenetic tree. Metadata and phylogenetic trees were visualised using iTOL v4 [24]. To identify the bacterial sequence types, AMR genes, virulence loci and plasmid replicon types, the assembled contigs were analysed by multilocus sequence typing (MLST)1.8 [25], ResFinder3.1.0 [26], the Comprehensive Antibiotic Research Database [27], Kleborate3.0 (https://github.com/katholt/Kleborate), and PlasmidFinder2.0 [28], respectively. Virulence loci (yersiniabactin, $y b t$; colibactin, $c l b$; salmochelin, iro; aerobactin, iuc; hypermucoidy, rmpA1, rmpA2; Klebsiella capsule Klocus, $\mathrm{KL}$; and ICEKp-associated virulence loci in CPKP) were identified using Kleborate v3.0 (https://github.com/ katholt/Kleborate). The virulence scores, which ranged from 0 to 5 , were calculated as follows: $0=$ no virulence loci; 1 = yersiniabactin only; 2 =yersiniabactin and colibactin, or colibactin only; $3=$ aerobactin and/or salmochelin only (without yersiniabactin or colibactin); $4=$ aerobactin and/or salmochelin with yersiniabactin (without colibactin); and $5=$ yersiniabactin, colibactin and aerobactin and/or salmochelin.

\section{Results}

\section{Clinical characteristics of the study participants}

Thirty-three patients had their CPE isolates whole genome sequenced for this study. Of these, 15 patients were found to be colonised with CPE, while the others had a confirmed CPE infection at the time of enrolment. Nineteen of the 33 patients (57.6\%) were female. The mean age of all the patients was 62.8 years (IQR, interquartile range: $47-81$ years) (Table 1 ). Most CPE-colonised patients $(n=13 / 15)$ had experienced prolonged hospitalisation before CPE was detected in their rectal swabs (median stay: 21 days, IQR 0-34 days), and 39\% of them were admitted to the intensive care unit. Thirty-two patients had underlying conditions such as diabetes mellitus or chronic kidney disease and had received antibiotic treatment within the month prior to enrolment, of which two thirds $(n=23)$ received carbapenems (Supplementary Table 1). Among the 15 CPE-colonised patients, three (16.7\%) had CPE colonisation detected prior to developing CPE infection during their hospital stay (median 16 days, IQR; 3-31.75 days) (Supplementary Table 1). We still included these three patients in the CPE-colonised group. Among the CPE-infected patients, ventilator-associated pneumonia (VAP) was the most common consequence of CPE infection, followed by urinary tract infection and primary bacteraemia (Supplementary Table 1). Only one CPE-infected patient received colistin monotherapy; all 17 of the other patients received colistin-based combination therapy, with the median duration of antibiotic treatment lasting 11 days 


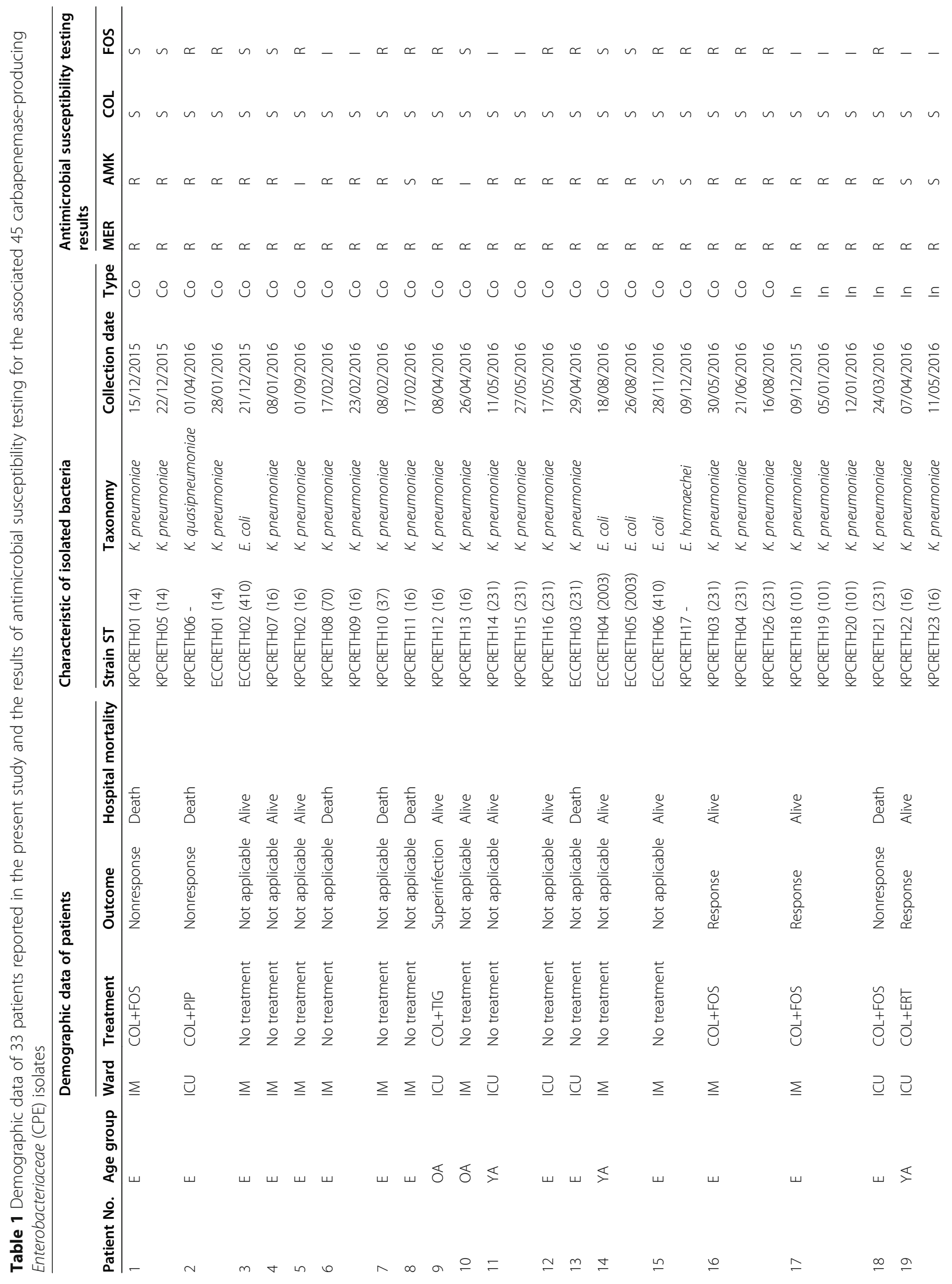




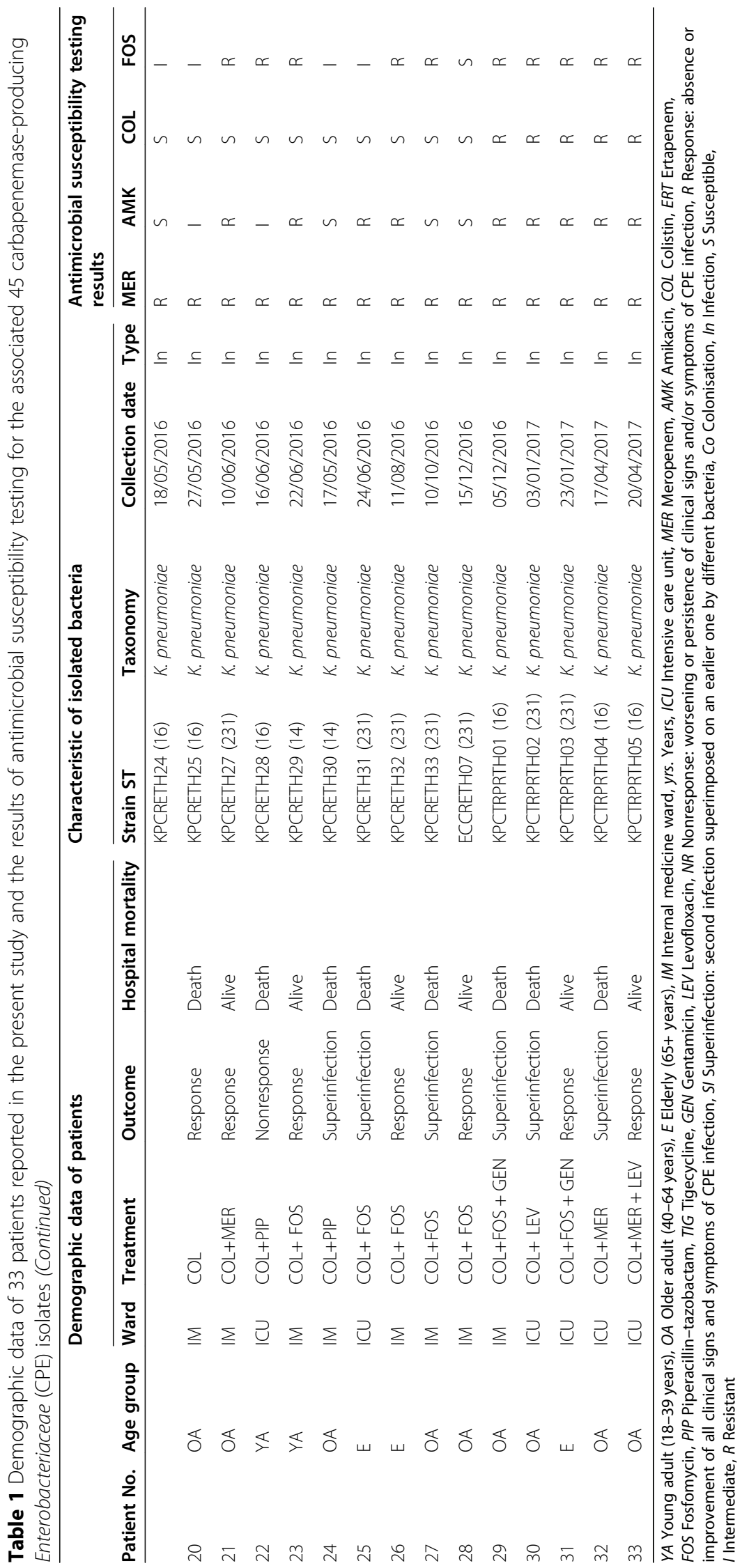


(IQR: 7-14 days) (Supplementary Table 1). Colistin-fosfomycin was the most common antimicrobial combination regimen $(45 \%, n=9 / 20)$ followed by colistin-piperacillin/tazobactam (15\%, $n=3 / 20)$ (Table 1). Colistin-fosfomycin was the first treatment option for patients with carbapenem-resistant infections because this combination has been shown to afford higher microbiological eradication rates than colistin monotherapy in Siriraj Hospital [29]. Regarding the local antibiogram, because $\mathrm{CPE}$ was more susceptible to piperacillin-tazobactam than to imipenem and meropenem, the second most common combination regime was colistin-piperacillin/tazobactam. Unfavourable clinical outcomes were observed in $52.4 \%$ of all the CPEinfected patients $(n=21)$, including three who were initially colonised with CPE but later developed CPE infections, and seven of these patients experienced superinfections with different bacterial species at the end of their antibiotic regimes (Table 1). There was no statistically significant mortality observed between the CPE-infected patients $(47.6 \%, n=10 / 21)$ vs. the CPE colonised ones $(33.3 \%, n=4 / 12$; chi-square test; $p=0.43)$.

\section{Antimicrobial susceptibility patterns detected in the CPE isolates}

We isolated 39 K. pneumoniae, four Escherichia coli, and one isolate each of Enterobacter hormaechei subsp. steigerwaltii and $K$. quasipneumoniae subsp. similipneumoniae from the 33 patients in our study (Table 1). All 45 isolates displayed meropenem resistance, only $20 \%$ of them $(n=9)$ were susceptible to amikacin, and $17.8 \%$ $(n=8)$ were susceptible to fosfomycin (Table 1). All of the CPE isolates were resistant to ciprofloxacin, cefoxitin, ceftriaxone, ceftazidime, piperacillin-tazobactam, ertapenem and imipenem (Table 1). Only five of the isolates, all $K$. pneumoniae, showed resistance to colistin with MIC values ranging between 32 and $64 \mathrm{mg} / \mathrm{L}$ (Table 1). No significant differences between the antimicrobial susceptibility patterns of isolates from CPEcolonised patients and those from CPE-infected patients were observed, indicating that colonising and diseasecausing strains show very similar AMR profiles, although this finding may also be attributed to the relatively small sample size available.

\section{High diversity in AMR genes and plasmids in the CPE isolates}

Our genomic analysis showed that bla $a_{\text {OXA-232 }}$ was the most dominant carbapenemase gene family and was found in 34 of $39 \mathrm{~K}$. pneumoniae and two of the four $E$. coli isolates we sequenced (Supplementary Table 2). The two most common sequence types (STs) identified in $K$. pneumoniae were ST16 $(n=15)$ and ST231 $(n=14)$, from which 12 ST16 isolates carried bla OXA-232 and bla $a_{\mathrm{NDM}-1}$, whereas almost all of the ST231 $(n=13)$ isolates carried only bla OXA-232 $_{2}$ (Fig. 1, Supplementary Table 2). In addition, all of the CPE isolates carrying $\beta$ lactamase genes also carried genes encoding other AMR genes, including aminoglycosides (aac(6)-I, aph [3]), fluoroquinolones ( $q n r \mathrm{~B}, q n r \mathrm{~S})$, and fosfomycins (fos A6, Uhp T) (Supplementary Table 2). None of the five colistin-resistant isolates harboured mor-genes, although they were highly resistant to colistin (Table 1), We found a pmrB (D150Y) mutation in KPCTRPRTH02 and KPCTRPRTH04, $m g r \mathrm{~B}$ disruptions were observed in KPCTRPRTH03 (W20*) and KPCTRPRTH01 (Q30*). In isolate KPCTRPRTH05, a S60L mutation in YcaR was also identified (Brinkac et al., manuscript in preparation).

We identified the following range of incompatibility (Inc) plasmid groups in the CPE isolates: FIA, FIB (pQil), FII, HI2B, N2 and R (Fig. 1). We were particularly interested in the presence of IncFIB and the small-sized Col plasmid group in our CPE dataset because these two plasmid groups are reported to be most commonly found in clinical samples and are associated with the spread of AMR genes [30]. Interestingly, in our dataset, all cases where ST231-CPKP was present $(n=14)$ and nearly half of those with ST16-CPKP $(n=7)$ contained an IncFIB (pQil)-like plasmid (Fig. 1). Additionally, genomic analysis indicated that all bla $a_{\mathrm{OXA}-232}$-containing $\mathrm{CPE}$ isolates were predicted to contain ColKp3 plasmid replicons (Fig. 1).

\section{K. pneumoniae isolates carry genes associated with hypervirulence}

We searched the $K$. pneumoniae genomes for the virulence genes previously found in hvKP strains, including those encoding siderophores for the biosynthesis and uptake of iron ( $y b t$, iuc and iro) and genes for the regulator of mucoid phenotype (rmpA1/rmpA2) [13]. The $y b t$ locus, encoding the siderophore yersiniabactin, was present in 38/39 of the CPKP genomes. The most common allele, $y b t 14$ (located on ICEKp5), was identified in 19 isolates, while the second most common allele, $y b t 9$ (located on ICEKp3), was identified in 17 isolates, and the rest two isolates had $y b t 8$ (located on ICEKp9) and $y b t 10$ (located on ICEKp4), respectively (Fig. 2). Notably, iuc5, encoding the siderophore aerobactin, was only detected in ST231 $(n=14)$. We detected six distinct K locus (KL) types among 39 CPKP isolates, the most frequent ones being KL51 $(n=28)$, KL2 $(n=5)$, and KL17 $(n=3)$ (Fig. 2). Virulence plasmid-associated loci such as iro, encoding the siderophore salmochelin, colibactin and rmpA1 and rmpA2 were not present in the investigated CPKP genomes. We also found that wzi50 was more common in the ST16 isolates, whereas wzi104 was 


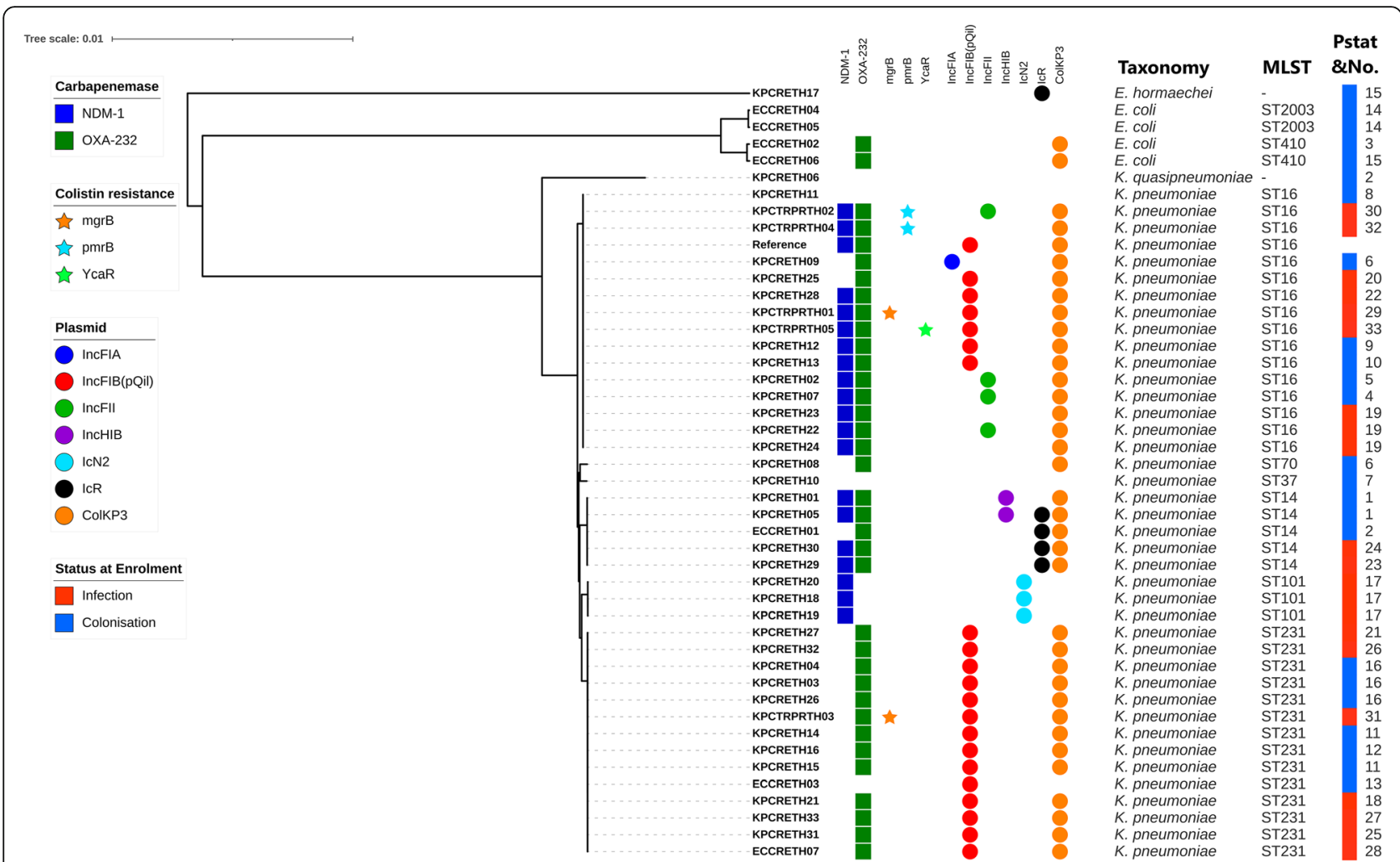

Fig. 1 A phylogenetic tree showing the relationship between all 45 carbapenemase-producing Enterobacteriaceae (CPE) genomes studied. The panel on the right shows the number of properties of the genomes-namely (from left to right), the presence (coloured symbol) and absence (no symbol) of the selected antimicrobial resistance genes (beta-lactamase genes: bla $a_{\mathrm{NDM}-1}$, bla $a_{\mathrm{OXA}-232 ;}$ colistin-resistant mutations present in: mgrB, pmrB, YcaR), plasmid replicons (circles), bacterial species, sequence type based on MLST profiles, patient's status at enrolment and patient number (Pstat\&No). The phylogenetic tree was based on 123,598 core SNPs of 45 CPE isolates using a reference genome (K. pneumoniae QS170029; NCBI no.: GCA_003073235.1). Scale bar indicates the number of nucleotide substitutions per site

only found in the ST231 isolates, and capsular antigen KL51 was found in ST16 and ST231 isolates alike.

\section{Associations between the patients' clinical data and the CPE isolates}

We identified nine patients who had more than one CPE isolate isolated throughout their hospital stay (Table 1). Six of them, despite receiving appropriate treatment, had $\geq$ 2 follow-up isolates that were the same bacterial species with the same sequence type and similar antibiogram pattern (Table 1). Genomic analysis also confirmed that the bacterial isolates from the same patient were identical with only $0-1$ single-nucleotide polymorphism (SNP) difference.

We noted that the bla OXA-232 carbapenemase-encoding ColKP3 plasmid was present in different strains of K. pneumoniae as well as in E. coli (Fig. 1), indicating the possibility of horizontal interspecies spread of this plasmid and possibly resulting in a polyclonal outbreak within our hospital. Although ST231 and ST16 were the two main clones associated with invasive disease and poor outcomes in our study, we did not identify any particular STs that were found only in CPE-colonised patients or only in CPE-infected patients.

\section{Discussion}

To the best of our knowledge, this is the first study to document detailed molecular bacterial isolate information on carbapenem resistance, plasmid replicons, and virulence determinants in relation to the clinical characterisation of hospitalised patients in Thailand. Of the 25 CPE-patients with follow-up rectal swab cultures, the mean time to culture negativity was 37.7 days in our study. This finding is consistent with previous reports that $54 \%$ of CPE rectal carriers remain CPE carriers for 30 to 60 days after their initial screening, 28\% remain as such after 6 months to 1 year, and 14\% remain as such after 1 year [31, $32]$. In our study, approximately $16.7 \%$ of the asymptomatic rectal carriers developed a clinical infection with a median duration of 20 days. The incidence of CPE infections in the $\mathrm{CPE}$-colonised patients in our study was as high as that seen previously [33,34], and there are several possible explanations for this. One explanation is that we began to observe patients who already had CPE colonisation at sites other than the gut, which might be a risk factor for them developing clinical infections [35]. Another explanation is that most of the patients had multiple comorbidities (e.g., 


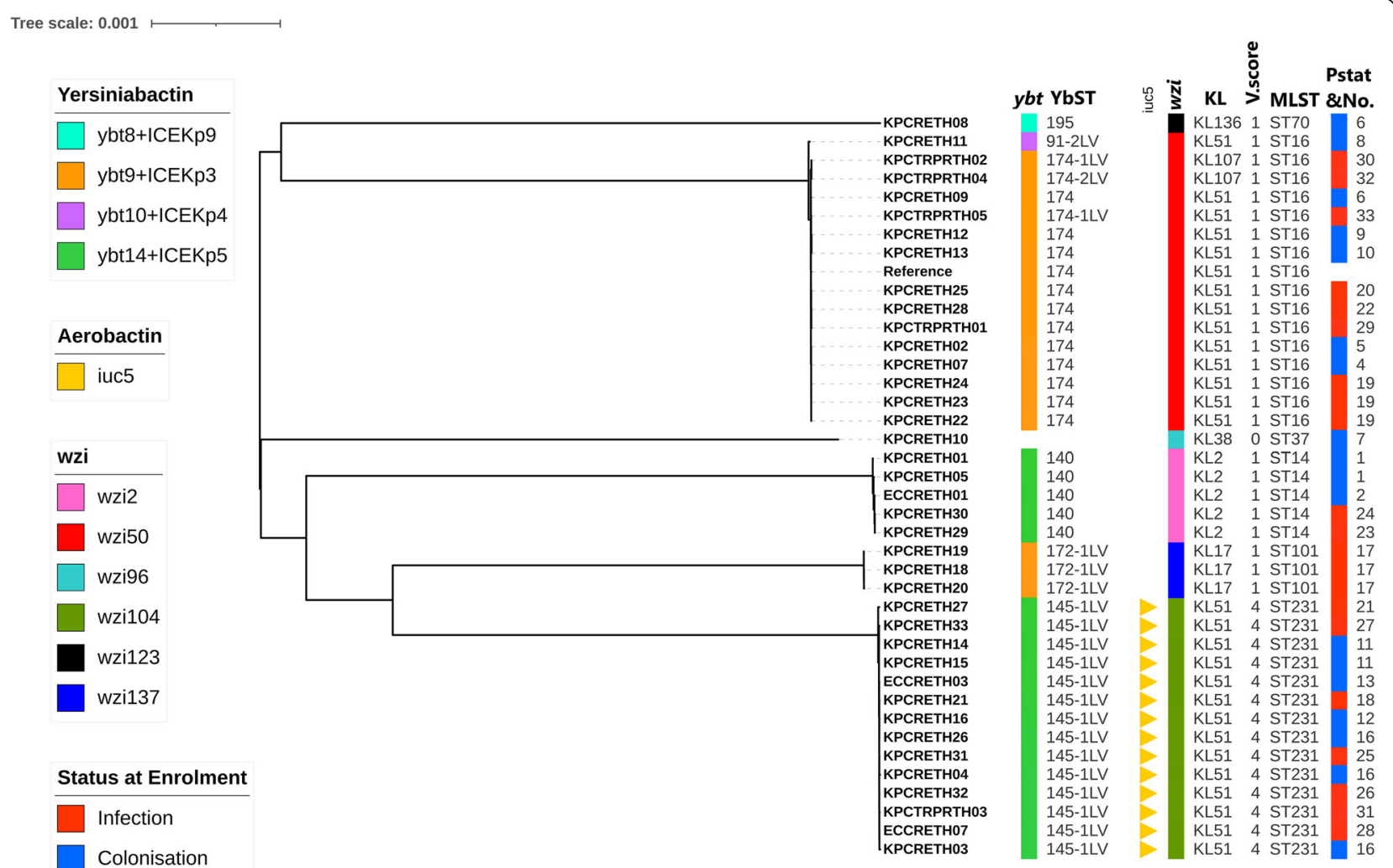

Fig. 2 A phylogenetic tree showing the relationship between 39 carbapenemase-producing Klebsiella pneumoniae (CPKP) isolates. The panel on the right shows a number of properties of the genomes-namely (from left to right), the presence or absence of virulence determinants such as ybt and ICEKp, ybt sequence type (YbST), iUc, wzi allele, KL and virulence score (V.score); sequence type based on MLST profiles; patient's status at enrolment and patient number (Pstat\&No). The phylogenetic tree is based on 70,571 core SNPs of the 39 CPKP isolates using a reference genome (K. pneumoniae QS17-0029; GCA_003073235.1). Scale bar indicates the number of nucleotide substitutions per site

diabetes mellitus and renal diseases) resulting in prolonged hospitalisation, possibly predisposing them to CPE colonisation and subsequent CPE infection.

Antimicrobial susceptibility testing in our study confirmed resistance to piperacillin/tazobactam, ciprofloxacin and meropenem in all the CPE isolates. Moreover, only $17.8 \%$ of the CPE strains isolated from the patients were susceptible to fosfomycin. Colistin is presumably the most active agent against up to $89 \%$ of the CPE isolates from our study. The evidence from a cohort study [26] and systematic review [13] on antibiotic therapy in CPE infections revealed that combination therapy is probably more effective than monotherapy. Therefore, the antibiotic therapy recommendation for CPE infections at Siriraj Hospital is combination therapy, with colistin acting as the backbone of the regimen.

In our study, the mortality rate was $47.6 \%$ for CPEinfected patients and $33.3 \%$ for patients colonised with CPE. The difference was not statistically significant. However, attributable mortality is difficult to assess because both groups already had high overall mortality and the sample size was small.
Although KPC-producing Enterobacteriaceae are reported to have spread rapidly over the last decade, their prevalence in Thailand remains very low [6, 7]. Notably, the CPE prevalence was $1.4 \%$, and $b l a_{\mathrm{KPC}-13}$ and $b l a_{\text {IMP-14 }}$ were the only carbapenemase genes detected among the CPE isolates identified at Siriraj Hospital during 2009 to 2011 [6]. However, the incidence of CPE bacteraemia has significantly increased from $<1 \%$ in 2011 to $3.8 \%$ in 2017 [6, 36]. The main CPE identified herein was CPKP, which carried one carbapenemase gene $\left(b l a_{\mathrm{NDM}-1}\right.$ or $\left.b l a_{\mathrm{OXA}-232}\right)$ and at least one other bla gene. $b l a_{\text {OXA-48- }}$-like genes were the most common carbapenemase genes, with bla OXA-232 detected in $78 \%$ of the isolates. We also found that $46 \%$ of the $b l a_{\text {OXA-232 }}$ isolates also carried $b l a_{\mathrm{NDM}-1}$, a finding consistent with that reported previously in Thailand [7]. This highlights that isolates with $b l a_{\mathrm{OXA}-48}$-like genes continue to be a problem in Thailand.

The previously reported cases of bla $a_{\mathrm{OXA}-232}$-harbouring $K$. pneumoniae were mainly serotypes ST14 and ST231 [37-39], while ST16 and ST231 were the dominant epidemic serotypes in our study. Thus, ST231 may 
be a high-risk, carbapenem-producing $K$. pneumoniae clone actively disseminating across Southeast Asia, with related outbreaks being reported in Switzerland [40]. We found that all 36 bla $a_{\text {OXA-232-harbouring isolates were }}$ present on a small ColKP3 plasmid in our dataset of $E$. coli and $K$. pneumoniae genomes, a finding concordant with that from a previous report [37]. Interestingly, IncFIB(pQil) plasmids were identified in all ST-231 $K$. pneumoniae isolates in our study, and both ColKP3 and IncFIB(pQil) are known to carry bla $a_{\mathrm{OXA}-232}$ and bla $a_{\mathrm{TEM}-1}$ $[41,42]$. These findings confirm that both plasmids, IncFIB(pQil) and ColKP3, are often found in clinical isolates and contain multiple AMR genes, as has been previously reported [30].

Among our CPKP isolates, we found two virulence loci that have been previously associated with invasive diseases: $y b t$ and $i u c$, encoding the siderophores yersiniabactin and aerobactin, respectively $[8,12,43]$. $Y b t$ was found in almost all of our CPKP isolates (97.4\%), and all $y b t$ loci detected in the CPKP genomes were associated with an ICEK $p$ structure located in a chromosomal region [12]. ICE $K p$, an integrative conjugative element, is self-transmissible and occasionally contains virulence factors such as $y b t$ and iro [12]. Thus, ICEKp is considered to be an important mediator of pathogenicity in $K$. pneumoniae [12]. VAP was the most common CPKP infection, and since all isolates from patients with VAP had $y b t$, these findings raise the interesting possibility that the yersiniabactin siderophore can promote respiratory tract infection as previous studies [44, 45]. This is the first identification of iuc5 in ST231-CPKP isolates in Thailand and Southeast Asia; otherwise, iuc5 has only been found in ST231-CPKP from India [46]. Some KL types (e.g., KL1, KL2, KL5 and KL57) are considered to be hypervirulent variants of $K$. pneumoniae and are associated with invasive diseases [47]. Our results show that there were at least seven distinct Klebsiella capsule genes/loci present among the 39 isolates, from which KL51 was the most common. However, only 5 out of 39 of our isolates were KL2 types and all of them belonged to ST14, a nonhypervirulent clone usually encountered in hospitalacquired infections [48]. Our results also revealed that wzi alleles were associated with the expected MLSTs more than with KL types.

Of note, when we integrated the clinical information with the bacterial genomic data, we identified ST231CPKP as the most common pathogen in CPE-infected patients, 6 out of 11 of which had invasive diseases such as primary bacteraemia and pneumonia. According to our analysis of virulence determinants, ST231-CPKP had the highest virulence score (Fig. 2) and contained iuc5. The iuc locus has been increasingly detected in hvKP over the last couple of years and is considered to be one of the most prominent features of invasive isolates [46, 49]. Second, four out of five of the colistin-resistant CPKP isolates belonged to ST16. ST16-CPKP with colistin resistance was found in $\mathrm{CPE}$-infected patients presenting with VAP and three of these patients died while in hospital. Therefore, ST16-CPKP is considered to be one of the more clinically significant clones in our study, as was also reported elsewhere [50]. Third, the core SNP-based phylogenetic tree suggests the possibility of a polyclonal outbreak of CPKP, predominantly involving ST231 and ST16 CPKP in Siriraj Hospital between 2015 and 2017. We identified two major subclades of CPKP: ST231 $(n=15)$ and ST16 $(n=14)$. Lastly, ST101 and ST14 were identified among the CPE-infected patients, something previously reported in South and Southeast Asia [46, 51].

Several limitations in our study require mentioning. First, the strains examined were all isolated from rectal swabs, and CPEs that caused infections were not characterised. However, we collected the rectal swabs while the patients were infected with CPEs and the antibiograms of CPE isolates at the sites of infection were similar to the antibiograms of CPE isolates from the rectal swabs. Next, it was not possible to identify the risk factors potentially associated with poor outcomes because of the small sample size that was available in this study. We have probably overestimated the true prevalence of CPE colonisation because there was a lack of routine screening for CPE in patients on admission during the study period. Nevertheless, our results suggest some clinical correlations between the clinical outcomes of patients with $\mathrm{CPE}$ infections and the genomic analysis of the organisms responsible, and also provide essential epidemiological data that could be used to guide empirical treatment and infection control strategies for CPE patients.

\section{Conclusion}

Our study represents the first report of a genomic epidemiological investigation on CPE among hospitalised patients in Thailand. By analysing resistance and virulence genes in combination with clinical patient information and bacterial genetic diversity, our approach provides important information that can be used to promptly track the emergence and spread of clinically significant isolates, suggest empirical antibiotics, assess mechanisms of drug resistance, and guide infection control strategies for CPE. Future largerscale studies are needed to determine the true prevalence of CPE and to identify the risk factors for CPE acquisition and their impact on treatment outcomes in Thailand. 


\section{Supplementary Information}

The online version contains supplementary material available at https:/doi. org/10.1186/s12879-021-05790-9.

Additional file 1: Table S1. Detailed clinical information of 33 carbapenemase-producing Enterobacteriaceae (CPE) patients described in the study. Table S2. Antimicrobial resistance determinants for 45 carbapenemase-producing Enterobacteriaceae isolates identified from whole genome sequencing analysis.

\section{Acknowledgements}

We greatly appreciate Prof. Xavier Didelot for his valuable advice. We also acknowledge the National Institute for Health Research Health Protection Research Unit (NIHR HPRU) in Healthcare Associated Infections and Antimicrobial Resistance at Imperial College London in partnership with Public Health England (PHE). We thank Sandra Cheesman, PhD, from Edanz Group (https://en-author-services.edanzgroup.com/) for editing a draft of this manuscript. The views expressed are those of the author(s) and not necessarily those of the NHS, the NIHR, the Department of Health or Public Health England. EJ is an Imperial College Research Fellow and acknowledges the funding from Rosetrees Trust and The Stoneygate Trust.

\section{Authors' contributions}

$A B, V T$, and DF conceived the study. $A B, L B C G$, and $K N$ undertook data analysis with input from $\mathrm{KL}$ and $\Pi$. EJ provided additional input into the framing of the results. $A B, E J, V T$, and DF produced the first draft of the manuscript. All authors contributed to the final draft. The authors read and approved the final manuscript

\section{Funding}

This work has been funded in whole or part with federal funds from the National Institute of Allergy and Infectious Diseases; National Institutes of Health; Department of Health and Human Services [U19Al110819]; and the Health Systems Research Institute (Thailand).

\section{Availability of data and materials}

Raw sequencing reads and assemblies are deposited in GenBank and the Sequence Read Archive under project accession: PRJNA389557. The biosample ID of each strain is shown in Supplementary Table 2.

\section{Ethics approval and consent to participate}

This study protocol was approved by the Siriraj Institutional Review Board (Si571/2015) with written informed consent obtained from all the participating patients.

\section{Consent for publication}

Not applicable.

\section{Competing interests}

None declared.

\section{Author details}

'Department of Research and Development, Faculty of Medicine, Siriraj Hospital, Mahidol University, Bangkok, Thailand. ${ }^{2} \mathrm{NIHR}$ Health Protection Research Unit in Healthcare Associated Infections and Antimicrobial Resistance, Imperial College, London, UK. ${ }^{3}$ Department of Infectious Disease Epidemiology, School of Public Health, Imperial College, London, UK. ${ }^{4}$ J. Craig Venter Institute, Rockville, MD, USA. ${ }^{5}$ Noblis, Reston, VA, USA. ${ }^{6}$ Department of Medicine, Faculty of Medicine Siriraj Hospital, Mahidol University, Bangkok, Thailand.

Received: 21 July 2020 Accepted: 11 January 2021 Published online: 04 February 2021

\section{References}

1. Temkin E, Adler A, Lerner A, Carmeli Y. Carbapenem-resistant Enterobacteriaceae: biology, epidemiology, and management. Ann N Y Acad Sci. 2014;1323:22-42.
2. Xu Y, Gu B, Huang M, Liu H, Xu T, Xia W, et al. Epidemiology of carbapenem resistant Enterobacteriaceae (CRE) during 2000-2012 in Asia. J Thorac Dis. 2015;7(3):376-85.

3. Center NARS. Antimicrobial Resistance 2000-2016; 2016.

4. Chotiprasitsakul D, Srichatrapimuk S, Kirdlarp S, Pyden AD, Santanirand P. Epidemiology of carbapenem-resistant Enterobacteriaceae: a 5-year experience at a tertiary care hospital. Infect Drug Resist. 2019;12:461-8.

5. Nordmann P, Naas T, Poirel L. Global spread of Carbapenemase-producing Enterobacteriaceae. Emerg Infect Dis. 2011;17(10):1791-8.

6. Netikul T, Kiratisin P. Genetic characterization of Carbapenem-resistant Enterobacteriaceae and the spread of Carbapenem-resistant Klebsiella pneumonia ST340 at a University Hospital in Thailand. PLoS One. 2015;10(9): e0139116.

7. Laolerd W, Akeda Y, Preeyanon L, Ratthawongjirakul P, Santanirand P. Carbapenemase-Producing Carbapenem-Resistant Enterobacteriaceae from Bangkok, Thailand, and Their Detection by the Carba NP and Modified Carbapenem Inactivation Method Tests. Microbial drug resistance (Larchmont, NY); 2018.

8. Holt KE, Wertheim H, Zadoks RN, Baker S, Whitehouse CA, Dance D, et al. Genomic analysis of diversity, population structure, virulence, and antimicrobial resistance in Klebsiella pneumoniae, an urgent threat to public health. Proc Natl Acad Sci U S A. 2015;112(27):E3574-81.

9. Munoz-Price LS, Poirel L, Bonomo RA, Schwaber MJ, Daikos GL, Cormican M, et al. Clinical epidemiology of the global expansion of Klebsiella pneumoniae carbapenemases. Lancet Infect Dis. 2013;13(9):785-96.

10. Durante-Mangoni E, Andini R, Zampino R. Management of carbapenemresistant Enterobacteriaceae infections. Clin Microbiol Infect. 2019;25(8):94350.

11. Bialek-Davenet S, Criscuolo A, Ailloud F, Passet V, Jones L, Delannoy-Vieillard AS, et al. Genomic definition of hypervirulent and multidrug-resistant Klebsiella pneumoniae clonal groups. Emerg Infect Dis. 2014;20(11):1812-20.

12. Lam MMC, Wick RR, Wyres KL, Gorrie CL, Judd LM, Jenney AWJ, et al. Genetic diversity, mobilisation and spread of the yersiniabactin-encoding mobile element ICEKp in Klebsiella pneumoniae populations. Microb Genom. 2018;4(9):e000196

13. Candan ED, Aksoz N. Klebsiella pneumoniae: characteristics of carbapenem resistance and virulence factors. Acta Biochim Pol. 2015;62(4):867-74.

14. Wyres KL, Holt KE. Klebsiella pneumoniae population genomics and antimicrobial-resistant clones. Trends Microbiol. 2016;24(12):944-56.

15. Institute CaLS. CLSI Supplement M100. 27th ed. Wayne: Clinical and Laboratory Standards Institute; 2017. Performance Standards for Antimicrobial Susceptibility Testing; 2017.

16. Institute CaLS. CLSI M07-A10 : Methods For Dilution Antimicrobial Susceptibility Tests For Bacteria That Grow Aerobically; Approved Standard -Tenth Edition. 2015.

17. Birgy A, Bidet P, Genel N, Doit C, Decre D, Arlet G, et al. Phenotypic screening of carbapenemases and associated beta-lactamases in carbapenem-resistant Enterobacteriaceae. J Clin Microbiol. 2012;50(4):1295302.

18. Bankevich A, Nurk S, Antipov D, Gurevich AA, Dvorkin M, Kulikov AS, et al. SPAdes: a new genome assembly algorithm and its applications to singlecell sequencing. J Comput Biol. 2012;19(5):455-77.

19. Tatusova T, DiCuccio M, Badretdin A, Chetvernin V, Nawrocki EP, Zaslavsky L, et al. NCBI prokaryotic genome annotation pipeline. Nucleic Acids Res. 2016; 44(14):6614-24.

20. Chavda KD, Chen L, Fouts DE, Sutton G, Brinkac L, Jenkins SG, et al. Comprehensive Genome Analysis of Carbapenemase-Producing Enterobacter spp.: New Insights into Phylogeny, Population Structure, and Resistance Mechanisms. mBio. 2016;7(6):e02093-16.

21. Brinkac LM, White R, D'Souza R, Nguyen K, Obaro SK, Fouts DE. Emergence of New Delhi Metallo- $\beta$-Lactamase (NDM-5) in Klebsiella quasipneumoniae from Neonates in a Nigerian Hospital. mSphere. 2019;4(2):e00685-18.

22. Srijan A, Margulieux KR, Ruekit S, Snesrud E, Maybank R, Serichantalergs O, et al. Genomic Characterization of Nonclonal mcr-1-Positive MultidrugResistant Klebsiella pneumoniae from Clinical Samples in Thailand. Microb Drug Resist (Larchmont, NY). 2018;24(4):403-10.

23. Price MN, Dehal PS, Arkin AP. FastTree: computing large minimum evolution trees with profiles instead of a distance matrix. Mol Biol Evol. 2009;26(7): 1641-50.

24. Letunic I, Bork P. Interactive tree of life (iTOL) v4: recent updates and new developments. Nucleic Acids Res. 2019;47(W1):W256-w9. 
25. Larsen MV, Cosentino S, Rasmussen S, Friis C, Hasman H, Marvig RL, et al. Multilocus sequence typing of total-genome-sequenced bacteria. J Clin Microbiol. 2012;50(4):1355-61.

26. Zankari $\mathrm{E}$, Hasman $\mathrm{H}$, Cosentino S, Vestergaard M, Rasmussen S, Lund O, et al. Identification of acquired antimicrobial resistance genes. J Antimicrob Chemother. 2012;67(11):2640-4

27. Jia B, Raphenya AR, Alcock B, Waglechner N, Guo P, Tsang KK, et al. CARD 2017: expansion and model-centric curation of the comprehensive antibiotic resistance database. Nucleic Acids Res. 2017:45(D1):D566-d73.

28. Carattoli A, Zankari E, Garcia-Fernandez A, Voldby Larsen M, Lund O, Villa L, et al. In silico detection and typing of plasmids using PlasmidFinder and plasmid multilocus sequence typing. Antimicrob Agents Chemother. 2014; 58(7):3895-903.

29. Sirijatuphat $R$, Thamlikitkul V. Preliminary study of colistin versus colistin plus fosfomycin for treatment of carbapenem-resistant Acinetobacter baumannii infections. Antimicrob Agents Chemother. 2014;58(9):5598-601.

30. Yan JJ, Ko WC, Wu JJ. Identification of a plasmid encoding SHV-12, TEM-1, and a variant of IMP-2 metallo-beta-lactamase, IMP-8, from a clinical isolate of Klebsiella pneumoniae. Antimicrob Agents Chemother. 2001;45(8):236871.

31. Feldman N, Adler A, Molshatzki N, Navon-Venezia S, Khabra E, Cohen D, et al. Gastrointestinal colonization by KPC-producing Klebsiella pneumoniae following hospital discharge: duration of carriage and risk factors for persistent carriage. Clin Microbiol Infect. 2013;19(4):E190-6.

32. Zimmerman FS, Assous MV, Bdolah-Abram T, Lachish T, Yinnon AM, WienerWell Y. Duration of carriage of carbapenem-resistant Enterobacteriaceae following hospital discharge. Am J Infect Control. 2013;41(3):190-4.

33. McConville TH, Sullivan SB, Gomez-Simmonds A, Whittier S, Uhlemann AC. Carbapenem-resistant Enterobacteriaceae colonization (CRE) and subsequent risk of infection and 90-day mortality in critically ill patients, an observational study. PLoS One. 2017;12(10):e0186195

34. Tischendorf J, de Avila RA, Safdar N. Risk of infection following colonization with carbapenem-resistant Enterobactericeae: a systematic review. Am J Infect Control. 2016:44(5):539-43.

35. Giannella M, Trecarichi EM, De Rosa FG, Del Bono V, Bassetti M, Lewis RE, et al. Risk factors for carbapenem-resistant Klebsiella pneumoniae bloodstream infection among rectal carriers: a prospective observational multicentre study. Clin Microbiol Infect. 2014;20(12):1357-62.

36. Sirijatuphat R, Sripanidkulchai K, Boonyasiri A, Rattanaumpawan P, Supapueng $\mathrm{O}$, Kiratisin $\mathrm{P}$, et al. Implementation of global antimicrobial resistance surveillance system (GLASS) in patients with bacteremia. PLoS One. 2018;13(1):e0190132.

37. Potron A, Rondinaud E, Poirel L, Belmonte O, Boyer S, Camiade S, et al. Genetic and biochemical characterisation of OXA-232, a carbapenemhydrolysing class D beta-lactamase from Enterobacteriaceae. Int J Antimicrob Agents. 2013;41(4):325-9.

38. Teo JWP, Kurup A, Lin RTP, Hsien KT. Emergence of clinical Klebsiella pneumoniae producing OXA-232 carbapenemase in Singapore. New Microbes New Infect. 2013;1(1):13-5.

39. Abdul Momin MHF, Liakopoulos A, Phee LM, Wareham DW. Emergence and nosocomial spread of carbapenem-resistant OXA-232-producing Klebsiella pneumoniae in Brunei Darussalam. J Glob Antimicrob Resist. 2017;9:96-9.

40. Mancini S, Poirel L, Tritten ML, Lienhard R, Bassi C, Nordmann P. Emergence of an MDR Klebsiella pneumoniae ST231 producing OXA-232 and RmtF in Switzerland. J Antimicrob Chemother. 2018:73(3):821-23.

41. Lutgring JD, Zhu W, de Man TJB, Avillan JJ, Anderson KF, Lonsway DR, et al. Phenotypic and genotypic characterization of Enterobacteriaceae producing Oxacillinase-48-like Carbapenemases, United States. Emerg Infect Dis. 2018; 24(4):700-9.

42. Martin J, Phan HTT, Findlay J, Stoesser N, Pankhurst L, Navickaite I, et al. Covert dissemination of carbapenemase-producing Klebsiella pneumoniae (KPC) in a successfully controlled outbreak: long- and short-read wholegenome sequencing demonstrate multiple genetic modes of transmission. J Antimicrob Chemother. 2017;72(11):3025-34

43. Brisse S, Passet V, Haugaard AB, Babosan A, Kassis-Chikhani N, Struve C, et al. Wzi gene sequencing, a rapid method for determination of capsular type for Klebsiella strains. J Clin Microbiol. 2013;51(12):4073-8.

44. Lawlor MS, O'Connor C, Miller VL. Yersiniabactin is a virulence factor for Klebsiella pneumoniae during pulmonary infection. Infect Immun. 2007; 75(3):1463-72.
45. Bachman MA, Oyler JE, Burns SH, Caza M, Lépine F, Dozois CM, et al. Klebsiella pneumoniae yersiniabactin promotes respiratory tract infection through evasion of lipocalin 2. Infect Immun. 2011;79(8):3309-16.

46. Wyres KL, Nguyen TNT, Lam MMC et al. Genomic surveillance for hypervirulence and multi-drug resistance in invasive Klebsiella pneumoniae from South and Southeast Asia. Genome Med. 2020;12:11. https:// genomemedicine.biomedcentral.com/articles/10.1186/s13073-019-0706y\#citeas.

47. Liu YM, Li BB, Zhang YY, Zhang W, Shen H, Li H, et al. Clinical and molecular characteristics of emerging hypervirulent Klebsiella pneumoniae bloodstream infections in mainland China. Antimicrob Agents Chemother 2014;58(9):5379-85.

48. Giske CG, Froding I, Hasan CM, Turlej-Rogacka A, Toleman M, Livermore D, et al. Diverse sequence types of Klebsiella pneumoniae contribute to the dissemination of blaNDM-1 in India, Sweden, and the United Kingdom. Antimicrob Agents Chemother. 2012;56(5):2735-8.

49. Russo TA, Olson R, Fang CT, Stoesser N, Miller M, MacDonald U, et al. Identification of Biomarkers for Differentiation of Hypervirulent Klebsiella pneumoniae from Classical K. pneumoniae. J Clin Microbiol. 2018;56(9): e00776-18.

50. Moradigaravand D, Martin V, Peacock SJ, Parkhill J. Evolution and Epidemiology of Multidrug-Resistant Klebsiella pneumoniae in the United Kingdom and Ireland. MBio. 2017;8(1):e01976-16.

51. Can F, Menekse S, Ispir P, Atac N, Albayrak O, Demir T, et al. Impact of the ST101 clone on fatality among patients with colistin-resistant Klebsiella pneumoniae infection. J Antimicrob Chemother. 2018;73(5):1235-41.

\section{Publisher's Note}

Springer Nature remains neutral with regard to jurisdictional claims in published maps and institutional affiliations.

Ready to submit your research? Choose BMC and benefit from:

- fast, convenient online submission

- thorough peer review by experienced researchers in your field

- rapid publication on acceptance

- support for research data, including large and complex data types

- gold Open Access which fosters wider collaboration and increased citations

- maximum visibility for your research: over $100 \mathrm{M}$ website views per year

At $\mathrm{BMC}$, research is always in progress.

Learn more biomedcentral.com/submission 